\title{
SEEN
}

\section{Evaluación de la eficacia y la seguridad de un protocolo de manejo de pacientes con diabetes descompensada por glucocorticoides durante la hospitalización}

\author{
Ana Agudo-Tabuenca ${ }^{\mathrm{a}, *}$, José Antonio Gimeno-Orna ${ }^{\mathrm{a}}$ y Daniel Sáenz-Abad ${ }^{\mathrm{b}}$ \\ a Instituto de Investigación Sanitaria Aragón (IIS Aragón), Servicio de Endocrinología y Nutrición, Hospital Clínico Universitario \\ Lozano Blesa, Zaragoza, España \\ ${ }^{\mathrm{b}}$ Instituto de Investigación Sanitaria Aragón (IIS Aragón), Servicio de Urgencias, Hospital Clínico Universitario Lozano Blesa, \\ Zaragoza, España
}

Recibido el 28 de octubre de 2018; aceptado el 15 de enero de 2019

\author{
PALABRAS CLAVE \\ Manejo de la \\ diabetes; \\ Hiperglucemia; \\ Glucocorticoides; \\ Diabetes esteroidea; \\ Protocolo terapéutico
}

\begin{abstract}
Resumen
Introducción: No existen protocolos consensuados de manejo hospitalario de las descompensaciones hiperglucémicas inducidas por dosis farmacológicas de glucocorticoides (GC). Nuestro objetivo fue evaluar la eficacia y la seguridad de un protocolo de insulinización específico para corticoides (PC) frente a un protocolo general (PG) en diabetes descompensada por GC (DDG). Materiales y métodos: Estudio experimental con grupo control, no aleatorizado, en pacientes con DDG ingresados en neumología. Se compararon 2 protocolos (PC y PG), ambos basados en terapia basal-bolo pero con diferentes dosis y distribución de insulina.

Se evaluó la diferencia de glucemia media (GM) durante la hospitalización entre el PC y el PG, así como el riesgo de presentar una $\mathrm{GM}>200 \mathrm{mg} / \mathrm{dl}$, ajustado para potenciales factores de confusión (relacionados con el paciente y con la terapia de GC empleada).

Resultados: Se incluyó a 131 pacientes, 60 asignados al PG y 71 al PC. Un 74\% de los pacientes estaban ingresados por exacerbación de enfermedad pulmonar obstructiva crónica. Hubo diferencia significativa en la dosis total de insulina entre el PG y el PC (29,4 vs. 57,4 unidades; $p$ < $0,0001)$.

La diferencia ajustada de GM (PC-PG) fue de $-14,8$ (IC del 95\%, -26,2 a -3,3) mg/dl. Los pacientes del PC tuvieron menor riesgo ajustado de presentar $\mathrm{GM}>200 \mathrm{mg} / \mathrm{dl}$ durante la hospitalización $(O R=0,31$; IC del 95\%, 0,11-0,91; $p=0,033)$. No hubo diferencias en el riesgo de hipoglucemia grave entre el PG y el PC ( $0 \%$ vs. $1,4 \%$; $p=0,36)$.

Conclusiones: El protocolo estudiado ha demostrado reducir la GM de pacientes con DDG durante la hospitalización sin comprometer su seguridad.
\end{abstract}

(c) 2019 SEEN y SED. Publicado por Elsevier España, S.L.U. Todos los derechos reservados.

\footnotetext{
* Autor para correspondencia.

Correo electrónico: a.agudota@gmail.com (A. Agudo-Tabuenca).
} 


\section{KEYWORDS}

Diabetes

management;

Hyperglycemia;

Glucocorticoids;

Steroid-induced

diabetes;

Management protocol
Assessment of the efficacy and safety of a protocol to manage glucocorticoid-induced hyperglycemia in diabetic patients during hospital stay

\begin{abstract}
Introduction: There are no agreed protocols on hospital management of hyperglycemic decompensation induced by pharmacological doses of glucocorticoids (GCs). The study objective was to assess the efficacy and safety of an insulin therapy protocol specific for patients treated with glucocorticoids (CP) as compared to a general protocol (GP) in diabetes decompensation secondary to glucocorticoids.

Materials and methods An experimental study in patients with glucocorticoids-induced decompensated diabetes admitted to a respiratory ward including a non-randomized control group. Two protocols (CP and GP), both based on basal-bolo insulin regimens, but with different insulin doses and distribution, were compared.

The difference in mean blood glucose (MBG) levels between both protocols was measured during hospital stay, as was the risk of having MBG levels $>200 \mathrm{mg} / \mathrm{dL}$, adjusted for potential confounding factors (related to patients and to the glucocorticoid therapy used).

Results: A total of 131 patients were included, 60 assigned to the GP and 71 to the CP groups. Seventy-four percent of patients had been admitted due to COPD exacerbation. There was a significant difference in the total daily insulin dose used between the CP and GP groups (29.4 vs. $57.4 \mathrm{IU} ; P<.0001)$.

The adjusted difference in MBG levels (CP-GP) was $-14.8(95 \% \mathrm{Cl},-26.2$ to -3.3$) \mathrm{mg} / \mathrm{dL}$. Patients in the CP group had a lower adjusted risk of having MBG levels $>200 \mathrm{mg} / \mathrm{dL}$ during hospital admission $(\mathrm{OR}=0.31 ; 95 \% \mathrm{Cl}, 0.11-0.91 ; P=.033)$. There were no differences in the risk of severe hypoglycemia between the CP and GP groups ( $0 \%$ vs. $1.4 \%$; $P=.36$ ).

Conclusions: The study protocol has been shown to decrease MBG levels in patients with glucocorticoids-induced decompensation of diabetes during hospital admission without compromising their safety.

(C) 2019 SEEN y SED. Published by Elsevier España, S.L.U. All rights reserved.
\end{abstract}

\section{Introducción}

Los glucocorticoides (GC), administrados a dosis suprafisiológicas, son agentes terapéuticos potentes utilizados en un gran número de enfermedades. Una de sus indicaciones más frecuentes en situaciones agudas es el tratamiento de la exacerbación de la enfermedad pulmonar obstructiva crónica $(\mathrm{EPOC})^{1-3}$.

Pese a ser fármacos de gran utilidad en la práctica clínica diaria, no están exentos de riesgos y efectos secundarios. El desarrollo de hiperglucemia en pacientes sin diagnóstico de diabetes previa, o la descompensación hiperglucémica en pacientes con diabetes conocida, es el más común de ellos. En un metaanálisis realizado por Liu et al. se comprobó que la incidencia de hiperglucemia entre todos los pacientes bajo tratamiento corticoideo era del $32,3 \%$, mientras que un $18,6 \%$ desarrollaba diabetes inducida por GC (DIG $)^{4}$. En un estudio que analizaba específicamente pacientes tratados con GC por enfermedad respiratoria, se halló una incidencia de DIG del $14,7 \%{ }^{5}$. Pese a todos estos datos, puede haber una infraestimación de la frecuencia real de DIG en dependencia de los criterios utilizados para su diagnóstico. Por el mecanismo de acción del GC (incremento de la resistencia insulínica con efecto predominantemente posprandial) y su utilización en muchos casos durante cortos periodos, es mejor basar el diagnóstico en la detección de glucemias $\geq 200 \mathrm{mg} / \mathrm{dl}$ en cualquier momento del día que en la glucemia en ayunas o en la $\mathrm{HbA} 1 \mathrm{c}^{1,6,7}$.

Se ha descrito una asociación entre el desarrollo de hiperglucemia y la aparición de complicaciones en el ingreso: aumento de infecciones nosocomiales, riesgo de desarrollar cetoacidosis diabética o síndrome hiperosmolar no cetósico, prolongación de la estancia hospitalaria y mayor mortalidad $^{1,2,8}$. Se ha llegado a establecer que por cada $18 \mathrm{mg} / \mathrm{dl}$ de aumento de glucemia hay un incremento de un $10 \%$ de la mortalidad ${ }^{9}$. En el momento actual, se define como control aceptable durante el ingreso hospitalario la consecución de glucemias medias entre 140 y $180 \mathrm{mg} / \mathrm{dl}^{10}$.

Los efectos sobre el metabolismo glucídico dependerán del tipo, la dosis y la pauta de administración del GC. En general, ante la administración de una dosis única matutina de metilprednisolona o de prednisona, el pico de hiperglucemia máximo se verá tras la comida de mediodía y antes de la cena, pudiendo ser normales los controles de la glucemia en ayunas. Sin embargo, en el caso de la utilización de dexametasona o dosis múltiples de metilprednisolona, la hiperglucemia será manifiesta de modo similar a lo largo de todo el día. Por ello, la pauta de dosificación de insulina deberá ser capaz de adaptarse al tipo y régimen de administración del GC y anticiparse a la modificación de las dosis del mismo, con el objetivo de conseguir un buen compromiso entre prevención de la hiperglucemia y de la 
hipoglucemia. Existen pocos datos en la literatura sobre cuál es la mejor aproximación terapéutica para conseguir un buen control glucémico hospitalario en pacientes tratados con dosis farmacológicas de GC. Aunque una glucemia media $(\mathrm{GM})<180 \mathrm{mg} / \mathrm{dl}$ durante el ingreso es definitoria de buen control glucémico, en pacientes complejos podrían aceptarse valores $<200 \mathrm{mg} / \mathrm{dl}^{11,12}$.

El objetivo de este estudio fue el de comprobar la eficacia y la seguridad de un nuevo protocolo de manejo de la hiperglucemia en pacientes con diabetes mellitus previa descompensada por GC (DDG) durante la hospitalización.

\section{Material y métodos}

\section{Diseño del estudio}

Estudio de intervención con grupo control, prospectivo, no aleatorizado, para evaluar la eficacia de un protocolo de tratamiento con insulina en pacientes con DDG durante su estancia hospitalaria. El estudio fue realizado entre el 4 de octubre del 2015 y el 6 de abril del 2017 en pacientes ingresados en la planta de Neumología de un hospital de tercer nivel. Hasta el 16 de octubre del 2016 se recogieron datos de los pacientes del grupo control y desde esta fecha hasta el cierre del estudio, datos de los pacientes asignados a la intervención. La duración de la intervención fue la misma que la del ingreso, si bien se recogieron únicamente los datos de los 15 primeros días de hospitalización.

\section{Consideraciones éticas}

A todos los pacientes se les ofreció consentimiento informado sobre el tratamiento que iban a recibir, explicándoles que era una modificación sobre el protocolo habitual intrahospitalario de administración de insulina. El estudio fue aprobado por el Comité Ético de Investigación Científica de Aragón (acta número 18/2014).

\section{Población del estudio}

Criterios de inclusión: diagnóstico previo de diabetes tipo 2 , edad mayor a 18 años, tratamiento con GC a una dosis inicial equivalente $\mathrm{a} \geq 0,5 \mathrm{mg} / \mathrm{kg} /$ día de metilprednisolona e ingreso previsto superior a 3 días.

Criterios de exclusión: gestantes, pacientes con filtrado glomerular (FG) inferior a $15 \mathrm{ml} / \mathrm{min} / 1,73 \mathrm{~m}^{2}$ y situación de cetoacidosis o síndrome hiperosmolar no cetósico al ingreso.

\section{Puntos finales clínicos}

Primario: GM durante el ingreso. Secundarios:

- Desviación estándar de todas las glucemias durante el ingreso.

- Riesgo de presentar GM durante la hospitalización > $180 \mathrm{mg} / \mathrm{dl}$.

- Riesgo de presentar GM durante la hospitalización > $200 \mathrm{mg} / \mathrm{dl}$.

\section{Variables recogidas}

Demográficas: edad, género, procedencia del paciente. Antropométricas: peso y talla, con cálculo del índice de masa corporal (IMC) como peso en $\mathrm{kg} /$ talla en $\mathrm{m}^{2}$.

Clínicas: antecedentes personales (hipertensión arterial, dislipidemia, enfermedad renal crónica, neoplasias, ictus, cardiopatía isquémica), tratamiento previo para la diabetes (codificado en 2 categorías según incluyese o no insulina), presión arterial al ingreso, índice de comorbilidad de Charlson, dosis total de insulina (DTI) diaria durante la hospitalización (basal y prandial), dosis máxima de insulina por kilogramo de peso y día alcanzadas, tipo y dosis de GC diarios (los diferentes tipos de GC se transformaron a su equivalente en miligramos de metilprednisolona por ser el más frecuentemente utilizado) y complicaciones durante el ingreso.

Analíticas: glucemia plasmática, creatinina (con cálculo del FG en $\mathrm{ml} / \mathrm{min} / 1,73 \mathrm{~m}^{2}$ mediante la fórmula de CKD-EPI) y $\mathrm{HbA1c}$ (obtenida durante el ingreso o en los 3 meses previos).

Relacionadas con el control de la glucemia durante el ingreso: a partir de los valores de glucemia capilar se determinaron como medidas de valoración del control glucémico intrahospitalario: glucemia máxima (el valor de glucemia capilar más elevado durante el ingreso), GM durante el ingreso, definida como la media de todas las glucemias capilares del paciente, desviación estándar (DE) de todas las glucemias capilares del paciente y coeficiente de variación, definido como el cociente entre la DE y la GM (DE/GM). Se consideró como buen control una $\mathrm{GM} \leq 180 \mathrm{mg} / \mathrm{dl}$ y como control aceptable una $\mathrm{GM} \leq 200 \mathrm{mg} / \mathrm{dl}$. Se registraron el número de hipoglucemias $(<70 \mathrm{mg} / \mathrm{dl}$ ) y su gravedad (se definió grave si era $<40 \mathrm{mg} / \mathrm{dl}$ o acompañada de pérdida de consciencia).

\section{Intervención sobre el control glucémico}

Grupo control. Al ingreso, se suspendieron todos los antidiabéticos orales (ADO) e insulinas premezcladas y se optó por tratamiento con pauta correctora, con insulina basal más pauta correctora o con tratamiento basal-bolo, siguiendo el protocolo general (PG) de manejo de la hiperglucemia implantado en nuestro hospital ${ }^{13}$. En ambos grupos las insulinas utilizadas fueron insulina glargina o detemir como insulinas de suplencia basal, e insulina aspart como insulina prandial y correctora. Cuando la insulina basal se administraba en 2 dosis era insulina detemir, y en caso de ser una sola dosis diaria, era insulina glargina. El médico neumólogo responsable del paciente podía aplicar el PG él mismo o solicitar colaboración con el servicio de Endocrinología. Se solicitó colaboración con Endocrinología en el 45\% de los pacientes del grupo control.

Grupo de intervención. A todos los pacientes del grupo experimental se les suspendieron todos los ADO y se les aplicó un nuevo protocolo de utilización de insulina adaptado al tratamiento con corticoides (PC) supervisado por un miembro del servicio de Endocrinología. Al igual que el PG, el PC estuvo basado en la utilización de una terapia basal-bolo, pero con dosis más elevadas de insulina y con 5 controles de glucemia capilar diarios (en ayunas, antes de comida, merienda y cena, y a medianoche) en vez de solo 3. 
En situación de ayuno, se administraba solo insulina basal + pauta correctora cada $4 \mathrm{~h}$. Ante un régimen normal de ingestas, se administraba el $50 \%$ de la DTI calculada como insulina basal en 2 dosis, y el otro $50 \%$ como bolos preprandiales, repartidos en desayuno (15\%), comida (15\%), merienda (10\%) y cena (10\%). Además, el PC permitía una adaptación flexible a las dosis cambiantes de GC durante la hospitalización. Si la administración de GC era en una sola dosis por la mañana, la insulina basal se administraba en una sola dosis coincidiendo con el momento de administración del GC, y los bolos preprandiales se repartían de diferente forma: un $10 \%$ en el desayuno, un $20 \%$ en la comida, un $15 \%$ en la merienda y un $5 \%$ en la cena. El objetivo era conseguir glucemias durante la hospitalización entre 100 y 200 mg/dl, dada la complejidad de alcanzar objetivos de control más estrictos en este grupo de pacientes.

Como tratamiento de rescate en ambos grupos, en caso de obtenerse un valor de glucemia por encima de 500, o 2 consecutivos por encima de $400 \mathrm{mg} / \mathrm{dl}$, se iniciaba una infusión continua de insulina por vía intravenosa.

El PG y el PC se adjuntan como anexo ( $A$ y $B$, respectivamente).

\section{Métodos de laboratorio}

La glucemia plasmática se determinó mediante método enzimático con hexocinasa, la HbA1c mediante cromatografía de alta resolución y la glucemia capilar se midió con medidor Optium Xceed $^{\circledast}$ (Abbott), cuya precisión es del 3-3,6\%, con una exactitud $r=0,98$ respecto a glucemia plasmática y un cumplimiento de la norma ISO del $99 \%$.

\section{Métodos estadísticos}

El tamaño muestral necesario para detectar una diferencia de GM clínicamente importante entre ambos grupos $(18 \mathrm{mg} / \mathrm{dl})$, con una potencia del $80 \%$ y un nivel de confianza del $95 \%$, asumiendo una DE de la GM de $35 \mathrm{mg} / \mathrm{dl}$, fue de 60 pacientes por grupo.

Las variables cuantitativas se describen con su media y $\mathrm{DE}$, mientras que las variables cualitativas se describen con su distribución de frecuencias. La comparación de variables cuantitativas entre los 2 grupos (PG y PC) se realiza con prueba no paramétrica de Mann-Whitney. La comparación de variables cualitativas se realiza mediante la prueba de la $\chi^{2}$ o test exacto de Fisher.

Debido a que no hubo aleatorización de la intervención, se procedió a calcular una estimación ajustada de la influencia del grupo de inclusión del paciente sobre el control glucémico intrahospitalario mediante regresión lineal multivariante. Las variables dependientes consideradas fueron la GM y la DE de todas las glucemias durante el ingreso, y los resultados se expresan en forma de diferencia ajustada (valores de glucosa [mg/dl] en PC - valores de glucosa $[\mathrm{mg} / \mathrm{dl}]$ en PG). El ajuste multivariante se realizó para las variables según su sentido clínico y estadístico (presencia de diferencias significativas entre los 2 grupos). Las variables de ajuste fueron edad, IMC, índice de Charlson, FG, glucemia inicial, $\mathrm{HbA} 1 \mathrm{C}$, tratamiento domiciliario para la diabetes, dosis media de GC y dosis máxima de GC por kilogramo de peso y día utilizada durante el ingreso.
El impacto de la utilización del PC sobre el riesgo de presentar durante el ingreso una GM > $180 \mathrm{mg} / \mathrm{dl}$ o una $\mathrm{GM}>200 \mathrm{mg} / \mathrm{dl}$ se evaluó mediante regresión logística multivariante ajustada para las mismas variables referidas anteriormente. Mediante procedimiento de exclusión secuencial se determinaron los principales factores predictores independientes de la adecuación del control glucémico hospitalario.

Se consideraron significativas las asociaciones con $\mathrm{p}<$ 0,05 . El programa informático utilizado fue el SPSS versión 22.0.

\section{Resultados}

\section{Descripción general de la muestra}

Se incluyó a un total de 131 pacientes (74\% varones), con una edad media de 72,2 (DE 11,2) años. El IMC medio fue de $30,6(D E 5,4) \mathrm{kg} / \mathrm{m}^{2}$, la puntuación media del índice de Charlson 3,44 (DE 1,9) puntos y el FG medio de 69,4 (DE $26,6) \mathrm{ml} / \mathrm{min} / 1,73 \mathrm{~m}^{2}$. Aproximadamente, la mitad de los pacientes $(50,4 \%)$ llevaba tratamiento domiciliario con insulina antes del ingreso y la HbA1c media fue de 7,7\% (DE $1,1)$.

Se asignó a 60 pacientes al grupo control con aplicación del PG y 71 al grupo de intervención con aplicación del PC. El motivo más frecuente de ingreso y tratamiento con GC fue exacerbación de EPOC (74\%), seguido de reagudización asmática $(13,7 \%)$. El GC más comúnmente utilizado fue la metilprednisolona por vía intravenosa, seguida de prednisona oral. Respecto a las dosis de GC que recibían los pacientes, no se encontraron diferencias estadísticamente significativas entre ambos grupos ni en la dosis media durante el ingreso $(56,9 \mathrm{mg} /$ día en el grupo control vs. $53,3 \mathrm{mg} /$ día en el grupo de intervención) ni en la dosis máxima por kilogramo de peso y día utilizada (1,29 vs. 1,15, respectivamente).

\section{Características de los pacientes según el grupo de asignación (tabla 1)}

Debido a la aplicación de los diferentes protocolos (PG y PC), aparecieron diferencias significativas entre el grupo control y el grupo de intervención en el número de glucemias diarias $(2,8$ vs. 3,$4 ; p<0,0001)$, en la proporción de pacientes en los que se realizó control de glucemia en la merienda ( 51,7 vs. $100 \%$; $p<0,0001)$, en las dosis medias de insulina basal (14,5 vs. 25,1 unidades; $p<0,0001)$, prandial $(14,9$ vs. 32,3 unidades; $p<0,0001)$ y total $(29,4$ vs. 57,4 unidades; $p<0,0001)$, en la dosis máxima de insulina alcanzada por kilogramo de peso y día (0,53 vs. 0,94 unidades; $p<$ 0,0001 ) y en el porcentaje de pacientes que al final del ingreso estaban en tratamiento basal-bolo (35 vs. $100 \%$; $p$ $<0,0001)$. Los pacientes del grupo control recibieron tratamiento con insulina programada tras llevar una media de 6 días de ingreso, mientras que en el grupo experimental ese tiempo se acortaba a 0,65 días $(p=0,001)$. 
Tabla 1 Características de los pacientes expresadas como media (desviación estándar) o como distribución de frecuencias (\%)

\begin{tabular}{lllll}
\hline Variable & Total & Protocolo general & Protocolo corticoides & p \\
\hline Género (\% varón) & 74 & 71,7 & 76,1 & 0,57 \\
Edad (años) & $72(11,2)$ & $72(11,8)$ & $72,4(10,7)$ & 0,86 \\
IMC (kg/m²) & $30,6(5,4)$ & $30,1(5,5)$ & $31(5,4)$ & 0,24 \\
Tratamiento con insulina en domicilio (\%) & 50,4 & 41,7 & 57,7 & 0,067 \\
Presión arterial sistólica (mmHg) & $144(26)$ & $142(26)$ & $145(25)$ & 0,55 \\
Índice de Charlson (puntuación) & $3,4(1,9)$ & $3,6(2,3)$ & $3,3(1,4)$ & 0,97 \\
Glucemia ingreso (mg/dl) & $212(80)$ & $225(87)$ & $201(72)$ & 0,16 \\
HbA1c (\%) & $7,7(1,2)$ & $7,5(1,1)$ & $7,9(1,2)$ & 0,069 \\
FG (ml/min/1,73 m²) & $69(27)$ & $69(27)$ & $70(26)$ & 0,81 \\
EPOC (\%) & 74 & 73,3 & 9,6 & 0,8 \\
Asma & 13,7 & 18,3 & $13(10,4)$ & 0,16 \\
Días de ingreso & $13,5(10,5)$ & $14,1(10,7)$ & $57,4(24)$ & 0,38 \\
DTI (unidades) & $44,5(26,7)$ & $29,4(21)$ & $25,1(11,7)$ & $<0,0001$ \\
DTI basal (unidades) & $20,3(13,8)$ & $14,5(14)$ & $32,3(14,7)$ & $<0,0001$ \\
DTI prandial (unidades) & $24,3(15,3)$ & $14,9(9,8)$ & $0,94(0,34)$ & $1,15(0,6)$ \\
Dosis máxima insulina/kg/día (unidades) & $0,76(0,4)$ & $0,53(0,4)$ & $53,3(22,5)$ & 0,0001 \\
Dosis máxima GC/kg/día (mg) & $1,21(0,6)$ & $1,29(0,7)$ & 0,0001 \\
Dosis media de glucocorticoide (mg/día) & $55(25)$ & $56,9(27,9)$ & 0,657 \\
\hline
\end{tabular}

DTI: dosis total de insulina; FG: filtrado glomerular; IMC: índice de masa corporal.

Tabla 2 Comparación de parámetros glucémicos entre ambos grupos

\begin{tabular}{|c|c|c|c|c|}
\hline Variable & Total & Protocologeneral & Protocolocorticoides & $\mathrm{p}$ \\
\hline Glucemia media desayuno (mg/dl) & $173,8(38,7)$ & $168,2(37)$ & $178,5(39,8)$ & 0,136 \\
\hline Glucemia media comida (mg/dl) & $213,9(44,6)$ & $229,5(41,5)$ & $200,8(43,1)$ & $<0,0001$ \\
\hline Glucemia media merienda (mg/dl) & $219,2(51,9)$ & $229,2(58,1)$ & $214,8(48,8)$ & 0,065 \\
\hline Glucemia media cena (mg/dl) & $191,9(49)$ & $210,6(54,6)$ & $176,1(37,3)$ & $<0,0001$ \\
\hline Glucemia media del ingreso $(\mathrm{mg} / \mathrm{dl})$ & $197,9(34)$ & $205,2(35,1)$ & $191,8(32)$ & 0,03 \\
\hline Número medio de controles glucémicos al día & $3,12(0,8)$ & $2,8(0,8)$ & $3,4(0,7)$ & $<0,0001$ \\
\hline Glucemia máxima durante el ingreso (mg/dl) & $361(75,8)$ & $365,5(81,7)$ & $357,1(70,9)$ & 0,51 \\
\hline Coeficiente de variación de glucemia durante ingreso (\%) & $35(8)$ & $34(9)$ & $36(7)$ & 0,131 \\
\hline DE glucemias durante ingreso $(\mathrm{mg} / \mathrm{dl})$ & 69,8 & 70,1 & 69,6 & 0,963 \\
\hline Media de hipoglucemias leves durante ingreso & $0,4(0,7)$ & $0,25(0,7)$ & $0,41(0,8)$ & 0,12 \\
\hline Media de hipoglucemias graves durante ingreso & 0,02 & 0 & 0,04 & 0,19 \\
\hline$\%$ hipoglucemias leves durante ingreso & 21,4 & 15 & 26,8 & 0,1 \\
\hline$\%$ hipoglucemias graves durante ingreso & 0,8 & 0 & 1,4 & 0,36 \\
\hline
\end{tabular}

Los valores se expresan como media (desviación estándar).

\section{Control glucémico hospitalario en dependencia del grupo de asignación (tabla 2)}

Glucemias medias diarias. A partir del segundo día de ingreso, las GM fueron inferiores en el grupo de intervención, siendo las diferencias estadísticamente significativas a partir del cuarto día (fig. 1). Una GM inferior a $180 \mathrm{mg} / \mathrm{dl}$ se alcanzó a partir del décimo día en el grupo control y a partir del quinto día en el grupo de intervención; una GM inferior a $200 \mathrm{mg} / \mathrm{dl}$ se alcanzó a partir del séptimo día en el grupo control y a partir del cuarto día en el grupo de intervención. Las GM fueron inferiores en el grupo de intervención en comida y cena, pero no hubo diferencias significativas ni en desayuno ni en merienda (fig. 2).

Diferencias ajustadas de GM y DE de todas las glucemias durante la hospitalización. Hubo diferencia significativa entre el grupo control y el grupo de intervención en la
GM (205,2 vs. 191,8 mg/dl; $p=0,030)$. La diferencia de GM, ajustada para potenciales factores de confusión, en el grupo de intervención frente al grupo control fue de $-14,8 \mathrm{mg} / \mathrm{dl}$ (IC del 95\%, -26,2 a -3,3) ( $p=0,012$ ). No hubo diferencia significativa entre el grupo control y el grupo de intervención en la DE $(70,1 \mathrm{vs.} 69,6 \mathrm{mg} / \mathrm{dl} ; \mathrm{p}=$ $0,96)$. La diferencia de DE, ajustada para potenciales factores de confusión, en el grupo de intervención frente al grupo control fue de $-1,5 \mathrm{mg} / \mathrm{dl}$ (IC del 95\%, -7,9 a 4,8) (p $=0,63)$.

Riesgo de presentar GM elevadas durante la hospitalización. Los pacientes del grupo de intervención tuvieron un riesgo ajustado más bajo, en el límite de la significación estadística, de presentar GM > $180 \mathrm{mg} / \mathrm{dl}$ durante la hospitalización (OR = 0,34; IC del 95\%, 0,10-1,15; $p=0,083$ ). Los pacientes del grupo de intervención tuvieron un riesgo ajustado más bajo, estadísticamente significativo, de presentar 
Glucemia media diaria a lo largo del ingreso

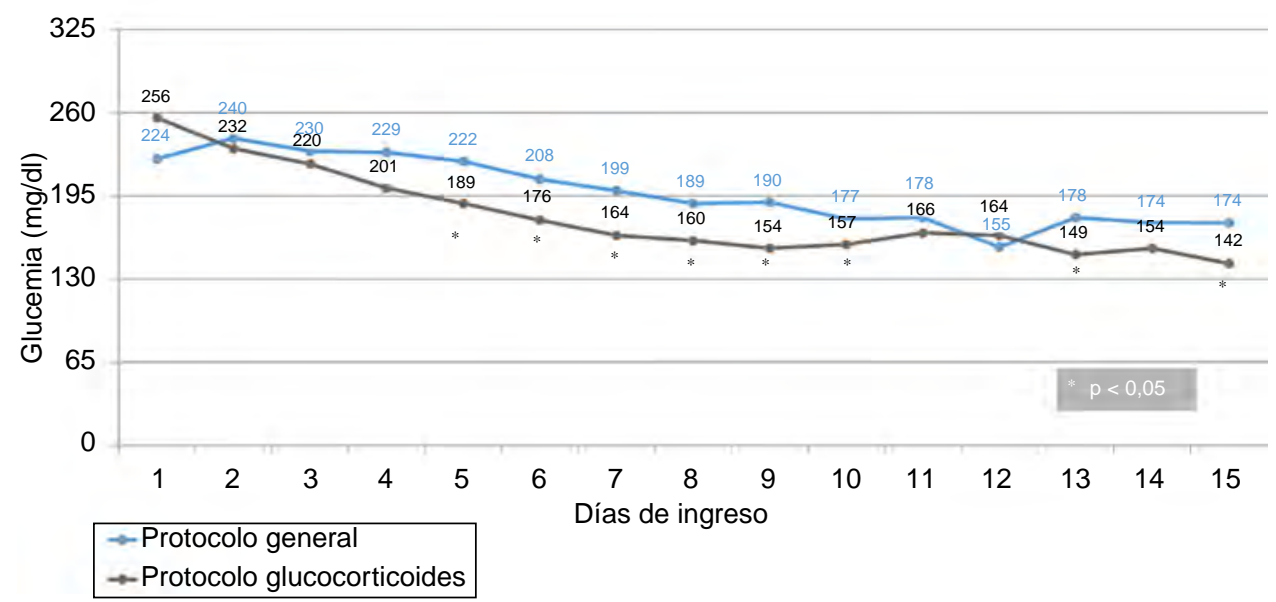

Figura 1 Glucemia media diaria a lo largo del ingreso. Los parámetros marcados con asterisco $\left({ }^{*}\right)$ representan los días con diferencias significativas en los parámetros de control glucémico entre ambos grupos.

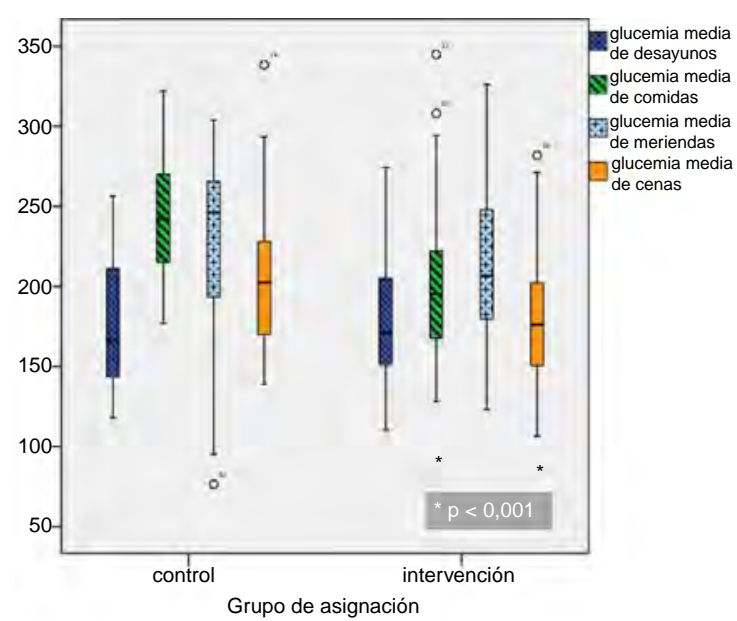

Figura 2 Glucemia diaria según el momento del día. Los parámetros marcados con asterisco $\left({ }^{*}\right)$ representan los días con diferencias significativas en los parámetros de control glucémico entre ambos grupos.

$\mathrm{GM}>200 \mathrm{mg} / \mathrm{dl}$ durante la hospitalización $(\mathrm{OR}=0,31$; IC del 95\%, 0,11-0,91; $p=0,033)$.

Factores predictores de $G M>200 \mathrm{mg} /$ dl durante la hospitalización. Mediante procedimiento de exclusión secuencial pudo determinarse que los factores predictores de presentar una $\mathrm{GM}>200 \mathrm{mg} / \mathrm{dl}$ fueron: asignación al grupo de intervención (factor protector con OR = 0,36; IC del 95\%, 0,15-0,86; $\mathrm{p}=0,018)$, una glucemia plasmática al ingreso más elevada $\left(\mathrm{OR}_{1 \mathrm{mg} / \mathrm{dl}}=1,007 ; \mathrm{IC}\right.$ del $\left.95 \%, 1,002-1,013 ; \mathrm{p}=0,010\right)$ y una HbA1c también más elevada $\left(\mathrm{OR}_{1 \%}=1,86\right.$; IC del $95 \%$, 1,24-2,77; $p=0,001)$.

\section{Complicaciones}

Las más frecuentes fueron infecciosas ( $5 \%$ grupo control vs. $0 \%$ grupo de intervención) y vasculares ( $5 \%$ vs. $7 \%$ ), sin diferencias significativas entre grupos. Hubo un total de 6 $(4,6 \%)$ pacientes que fallecieron durante la realización del estudio, $4(6,7 \%)$ en el grupo control y $2(2,8 \%)$ en el grupo de intervención, una diferencia que tampoco fue significativa. Los motivos de fallecimiento se consideraron en todos los casos derivados de la enfermedad que había condicionado el ingreso sin relación con el protocolo utilizado.

No se observaron complicaciones agudas de cetoacidosis diabética ni de síndrome hiperosmolar no cetósico. En cuanto al riesgo de hipoglucemia, no hubo diferencias significativas entre los 2 grupos: hipoglucemia leve en un 15\% del grupo control vs. un $26,8 \%$ del grupo de intervención ( $p$ $=0,1)$; hipoglucemia grave $0 \%$ vs. $1,4 \%(p=0,36)$.

\section{Discusión}

En el presente trabajo hemos podido comprobar que, mediante la aplicación de un protocolo específico de insulinización (PC) de pacientes con DDG, la GM durante el ingreso podía reducirse en aproximadamente $15 \mathrm{mg} / \mathrm{dl}$ sin comprometer la seguridad del paciente. La utilización del PC reducía en más de un $60 \%$ el riesgo de presentar GM superiores a $200 \mathrm{mg} / \mathrm{dl}$ durante la hospitalización.

La aplicación de protocolos específicos ha conseguido valores de GM muy variables, lo que puede deberse a varios factores, fundamentalmente relacionados con los criterios de inclusión de pacientes y con la terapia de GC empleada. En cuanto al perfil de pacientes incluidos, los estudios que reclutaban sujetos sin y con diabetes previa (una mezcla de pacientes con DIG y DDG) obtenían valores de GM más bajos: Lakhani et al. describieron en su estudio, con una prevalencia de DDG del 42\%, una GM en el grupo experimental de $170 \mathrm{mg} / \mathrm{dl}^{14}$. En cuanto al tipo y la dosis de GC utilizados, las GM son superiores en aquellos estudios, como el de Gosmanov et al., realizados sobre muestras de pacientes con procesos malignos hematológicos que requieren tratamiento con GC potentes de acción prolongada, como es la dexametasona (GM en el grupo asignado a tratamiento basal-bolo de $219 \mathrm{mg} / \mathrm{dl})^{15}$.

En nuestro trabajo obtuvimos con el PC una GM de $192 \mathrm{mg} / \mathrm{dl}$, frente a una GM de $205 \mathrm{mg} / \mathrm{dl}$ en el grupo tratado con el PG. Ruiz de Adana et al. ${ }^{16}$ consiguieron una GM 
de $205 \mathrm{mg} / \mathrm{dl}$ en el grupo asignado a tratamiento basal-bolo, con una dosis de insulina por $\mathrm{kg}$ de peso de 0,64 unidades. Este dato refuerza el mensaje de que es fundamental la dosis de insulina alcanzada, ya que en nuestro PC llegó a ser de 0,94 unidades por kilogramo de peso y día.

Existen pocos estudios que hayan analizado diferentes pautas de tratamiento de la DDG en pacientes ingresados en el hospital, y en nuestra revisión de la literatura no hemos hallado ninguno que evaluase prospectivamente los resultados completos durante un horizonte temporal de 2 semanas. El estudio de Lakhani et al. recogía los datos a lo largo del ingreso, si bien descartaba todos los valores de glucemia capilar de los días con glucemias $<70 \mathrm{mg} / \mathrm{dl}$ o $>400 \mathrm{mg} / \mathrm{dl}$, lo que podría no describir fielmente la realidad del ingreso ${ }^{14}$. En el estudio de Grommesh et al. se analizaban los resultados sin tener en cuenta los valores de glucemia capilar del día 1, que es un día en el que los valores de ambos grupos son elevados, debido probablemente a la falta de tiempo para que comience a funcionar el protocolo ${ }^{17}$; de hecho, en nuestro trabajo, no encontramos diferencias significativas en los valores de GM entre los grupos hasta el cuarto día. El estudio de Dhital et al. tenía un diseño retrospectivo, con análisis de los valores de glucemia capilar de un único día de ingreso ${ }^{18}$.

Existen varios protocolos publicados que defienden el manejo de la DIG o de la DDG mediante el uso de insulina $\mathrm{NPH}$. Aunque se ha aducido que el uso de NPH podría asociarse a un menor riesgo de hipoglucemias frente a insulina glargina o detemir ${ }^{17}$, en nuestro estudio, basado en una terapia basal-bolo, solo hubo una hipoglucemia grave en el grupo del PC, a pesar de las altas dosis de insulina administrada. Radhakutty et al., en su análisis de control glucémico los días 1 y 3 del ingreso aplicando una pauta basal-bolo versus insulina NPH, no observaron diferencias significativas ni en el control glucémico ni en la incidencia de hipoglucemias ${ }^{19}$. Otros estudios también han encontrado una eficacia similar entre NPH y pauta basal-bolo ${ }^{16,18}$. En nuestro estudio se usó una pauta bolo-basal diferente de la recomendada por Perez et al. ${ }^{1}$ para minimizar el riesgo de hipoglucemias, desplazando parte de la insulina de vespertina a la merienda.

Los resultados de nuestro estudio contribuyen a resaltar la eficacia y la seguridad de una pauta basal-bolo adaptada a la modificación de la dosis de GC. La diferencia ajustada de casi $15 \mathrm{mg} / \mathrm{dl}$ está en el límite de la relevancia clínica y no hubo diferencias significativas en el número de hipoglucemias. Creemos que la utilización de insulina NPH presenta varias desventajas respecto al tratamiento basal-bolo con análogos de insulina. En primer lugar, su perfil farmacodinámico más imprevisible, con picos de acción máxima difíciles de solapar con el pico de glucemia posprandial. En segundo lugar, en nuestro medio hospitalario las insulinas de uso más habitual (recogidas en el PG implantado) son glargina, detemir y aspart, aplicadas en terapia basal + dosis correctoras o en terapia basal-bolo; utilizar un protocolo con insulina NPH, con la que no está familiarizado el personal del hospital y que no ha demostrado mayor eficacia ${ }^{20,21}$, podría dificultar su implantación y manejo.

Contrariamente a algunos estudios publicados, en este estudio se suspendieron todos los ADO en el momento del ingreso. No defendemos mantenerlos dada la situación aguda y potencialmente grave del paciente ingresado, así como la posibilidad de desarrollar complicaciones derivadas de algunos de ellos, y el posible enmascaramiento de los resultados del protocolo evaluado. Así, en el estudio de Brady et al. al mantener todos los antidiabéticos o introducir metformina al ingreso si no la llevaban, resulta difícil discriminar qué parte del control conseguido se debe al protocolo estudiado o a los $\mathrm{ADO}^{22}$.

Las ventajas del presente estudio son el haber podido evaluar la eficacia y la seguridad de un nuevo modelo dinámico de insulinización en pacientes con DDG durante la estancia en el hospital, dada la falta de protocolos publicados en esta situación clínica. Dado su diseño prospectivo se pudo realizar una recogida exhaustiva de las glucemias de los pacientes. La aplicación del PC fue en todos los casos ejecutada por la misma persona, garantizando la homogeneidad de la intervención. Además, el número de pacientes incluidos fue superior al de otros estudios similares, teniendo un poder estadístico suficiente para detectar diferencias de GM clínicamente importantes.

Como limitaciones, cabe destacar fundamentalmente la falta de aleatorización de los pacientes; no obstante, no hubo diferencias entre los grupos excepto en variables relacionadas con el diferente protocolo utilizado (DTI, número de glucemias) y se realizó adicionalmente un análisis ajustado para los potenciales factores de confusión, por lo que consideramos que las diferencias en control glucémico observadas son una consecuencia de la aplicación del PC frente al PG. En segundo lugar, aunque el número de pacientes fue limitado, tuvimos suficiente potencia estadística para detectar diferencias en el control glucémico clínicamente relevantes; no obstante, el número de hipoglucemias leves fue superior en el grupo de intervención, por lo que no puede descartarse que con más pacientes la diferencia pudiese llegar a ser significativa. En tercer lugar, la diferencia a las indicaciones del $P C$ en cuanto al número de glucemias diarias no fue perfecta, lo que puede limitar su eficacia. Finalmente, el PC fue siempre implementado por la misma persona experta en el manejo de DDG, lo que podría haber influido positivamente en su utilidad; sería necesario evaluar su eficacia cuando se plantee su utilización por profesionales con diferente grado de experiencia. De hecho, en el grupo PG el retraso de 6 días hasta el inicio de insulina programada podría atribuirse a un cierto grado de inercia terapéutica.

En conclusión, la aplicación de un nuevo PC es eficaz y segura para abordar el frecuente problema de la hiperglucemia hospitalaria en pacientes tratados con GC. Consideramos nuestro trabajo como una prueba piloto de un PC que, si es validado y perfeccionado por otros profesionales y en otros hospitales, podría conducir al desarrollo de una forma consensuada de actuación ante la DDG.

\section{Autoría}

Los 3 autores citados han participado en la concepción y diseño del estudio así como en la recogida de datos. Además, han contribuido al análisis estadístico e interpretación de datos y todos ellos han revisado y aprobado el manuscrito remitido finalmente. 


\section{Financiación}

La presente investigación no ha recibido ayudas específicas provenientes de agencias del sector público, sector comercial o entidades sin ánimo de lucro.

\section{Conflicto de intereses}

Ninguno.

\section{Anexo. Material adicional}

Se puede consultar material adicional a este artículo en su versión electrónica disponible en doi:10.1016/j.endinu. 2019.01.008.

\section{Bibliografía}

1. Perez A, Jansen-Chaparro S, Saigi I, Bernal-Lopez MR, Miñambres I, Gomez-Huelgas R. Glucocorticoid-induced hyperglycemia. J Diabetes. 2014;6(1):9-20, http://dx.doi.org/10. 1111/1753-0407.12090.

2. Bonaventura A, Montecucco F. Steroid-induced hyperglycemia: An underdiagnosed problem or clinical inertia? A narrative review. Diabetes Res Clin Pract. 2018;139:203-20, http://dx.doi.org/10.1016/j.diabres.2018.03.006.

3. Hwang JL, Weiss RE. Steroid-induced diabetes: A clinical and molecular approach to understanding and treatment. Diabetes Metab Res Rev. 2014;30(2):96-102, http://dx.doi.org/10. 1002/dmrr.2486.

4. Liu XX, Zhu XM, Miao Q, Ye HY, Zhang ZY, Li YM. Hyperglycemia induced by glucocorticoids in nondiabetic patients: A meta-analysis. Ann Nutr Metab. 2014;65(4):324-32, http://dx.doi.org/10.1159/000365892.

5. Kim SY, Yoo CG, Lee CT, Chung HS, Kim YW, Han SK, et al. Incidence and risk factors of steroid-induced diabetes in patients with respiratory disease. J Korean Med Sci. 2011;26(2):264-7, http://dx.doi.org/10.3346/jkms.2011.26.2.264.

6. Uzu T, Harada T, Sakaguchi M, Kanasaki M, Isshiki K, Araki $S$, et al. Glucocorticoid-induced diabetes mellitus: Prevalence and risk factors in primary renal diseases. Nephron-Clin Pract. 2007;105(2):15-8, http://dx.doi.org/10.1159/000097598.

7. Iwamoto T, Kagawa $Y$, Naito $Y$, Kuzuhara S, Kojima M. Steroid-induced diabetes mellitus and related risk factors in patients with neurologic diseases. Pharmacotherapy. 2004;24(4):508-14, http://dx.doi.org/10.1592/phco.24.5.508. 33355.

8. Umpierez GE, Isaacs SD, Bazargan N, You X, Thaler LM, Kitabchi AE. Hyperglucemia: An independent marker of in-hospital mortality in patients with undiagnosed diabetes. J Clin Endocrinol Metab. 2002;87(3):978-82, http://dx.doi.org/10.1210/ jcem.87.3.8341.

9. Baker EH, Janaway CH, Philips BJ, Brennan AL, Baines DL, Wood $\mathrm{DM}$, et al. Hyperglycaemia is associated with poor outcomes in patients admitted to hospital with acute exacerbations of chronic obstructive pulmonary disease. Thorax. 2006;61(4):284-9, http://dx.doi.org/10.1136/thx.2005.051029.

10. American Diabetes Association. Diabetes Care in the hospital: Standards of medical care in diabetes-2018. Diabetes Care. 2018;41(Suppl 1):S144-S151. doi:10.2337/dc18 -S014.
11. Baldwin D, Apel J. Management of hyperglycemia in hospitalized patients with renal insufficiency or steroid-induced diabetes. Curr Diab Rep. 2013;13(1):114-20, http://dx.doi.org/10.1007/ s11892-012-0339-7.

12. Umpierrez GE, Hellman R, Korytkowski MT, Kosiborod M, Maynard GA, Montori VM, et al. Management of hyperglycemia in hospitalized patients in non-critical care setting: An endocrine society clinical practice guideline. J Clin Endocrinol Metab. 2012;97(1):16-38, http://dx.doi.org/10.1210/jc.2011-2098.

13. Sáenz-Abad D, Gimeno-Orna JA, Sierra-Bergua B, Lahoza-Pérez MC, Pérez-Calvo Jl. Evaluación de la eficacia de un protocolo destinado a mejorar el control glucémico de los pacientes con hiperglucemia ingresados en servicios hospitalarios de medicina interna. An Sist Sanit Navar. 2015;38(3):397-408.

14. Lakhani O, Kumar S, Tripathi S, Desai M, Seth C. Comparison of two protocols in the management of glucocorticoidinduced hyperglycemia among hospitalized patients. Indian J Endocrinol Metab. 2017;21(6):836, http://dx.doi.org/10.4103/ ijem.IJEM_226_17.

15. Gosmanov A, Goorha S, Stelts S, Peng L, Umpierrez G. Management of hyperglycemia in diabetic patients with hematologic malignancies during dexamethasone therapy. Endocr Pract. 2013;19(2):231-5, http://dx.doi.org/10.4158/EP12256.OR.

16. Ruiz de Adana MS, Colomo N, Maldonado-Araque C, Fontalba MI, Linares F, García-Torres F, et al. Randomized clinical trial of the efficacy and safety of insulin glargine vs. NPH insulin as basal insulin for the treatment of glucocorticoid induced hyperglycemia using continuous glucose monitoring in hospitalized patients with type 2 diabetes and respi. Diabetes Res Clin Pract. 2015;110(2):158-65, http:// dx.doi.org/10.1016/j.diabres.2015.09.015.

17. Grommesh B, Lausch MJ, Vannelli AJ, Mullen DM, Bergenstal RM, Richter SA, et al. Hospital insulin protocol AIMS for glucose control in glucocorticoid-induced hyperglycemia. Endocr Pract. 2016;22(2):180-9, http://dx.doi.org/10.4158/EP15818.OR.

18. Dhital S, Shenker Y, Meredith M, Davis D. A retrospective study comparing neutral protamine hagedorn insulin with glargine as basal therapy in prednisone-associated diabetes mellitus in hospitalized patients. Endocr Pract. 2012;18(5):712-9, http://dx.doi.org/10.4158/EP11371.OR.

19. Radhakutty A, Stranks JL, Mangelsdorf BL, Drake SM, Roberts GW, Zimmermann AT, et al. Treatment of prednisoloneinduced hyperglycaemia in hospitalized patients: Insights from a randomized, controlled study. Diabetes Obes Metab. 2017;19(4):571-8, http://dx.doi.org/10.1111/dom.12859.

20. Rys P, Wojciechowski P, Rogoz-Sitek A, Niesyczynski G, Lis J, Syta $A$, et al. Systematic review and meta-analysis of randomized clinical trials comparing efficacy and safety outcomes of insulin glargine with NPH insulin, premixed insulin preparations or with insulin detemir in type 2 diabetes mellitus. Acta Diabetol. 2015;52(4):649-62, http://dx.doi.org/10.1007/ s00592-014-0698-4.

21. Bazzano LA, Lee LJ, Shi L, Reynolds K, Jackson JA, Fonseca V. Safety and efficacy of glargine compared with NPH insulin for the treatment of type 2 diabetes: A meta-analysis of randomized controlled trials. Diabet Med. 2008;25(8):924-32, http://dx.doi.org/10.1111/j.1464-5491.2008. 02517.x.

22. Brady V, Thosani S, Zhou S, Bassett R, Busaidy NL, Lavis V. Safe and effective dosing of basal-bolus insulin in patients receiving high-dose steroids for hyper-cyclophosphamide, doxorubicin, vincristine, and dexamethasone chemotherapy. Diabetes Technol Ther. 2014;16(12.):874-9, http://dx.doi.org/10.1089/ dia.2014.0115. 